\title{
Acreditaciones y coaching en los Colegios Oficiales de Psicología de España: estado de la cuestión
}

\section{Accreditations and coaching in the Official Schools of Psychologists of Spain: state of the question}

Fecha de recepción: 31-10-2017

Fecha de aceptación: 11-05-2018

\author{
Alejo García-Naveira Vaamonde \\ Área I+D+i MAD Lions E.C.
}

\section{resumen/alostract:}

Los objetivos del presente trabajo son analizar la actual estructura de acreditación y los criterios de acreditación en coaching debido a que se percibe ciertas cuestiones a resolver por parte de los Colegios Oficiales de Psicología de España. Realizando una reflexión teórica sobre la temática, en cuanto a la estructura de las acreditaciones, no queda bien delimitado cuales representan áreas de conocimiento e intervención (especialidades) frente a las sub-áreas, ámbitos de actuación o estrategias de intervención (sub-especialidades). Respecto a las acreditaciones en coaching se observa una variedad de criterios establecidos y una falta de consenso entre los colegios de psicología, en el que además, se presenta como algo transversal y no relacionado a las especialidades. Como más destacable, se concluye que la estructura de acreditación tiene que quedar configurada con un primer nivel con el grado de psicología, un segundo nivel con la especialidad profesional y un tercer nivel de experto, vinculada con el nivel 2. En cuanto a la acreditación de coaching, se propone la denominación de psicólogo/a experto en coaching (nivel 3), un mínimo de 100 horas de formación y 100 horas de prácticas, superar una entrevista personal y prueba situacional, y renovación cada 4 años.

The objectives of this study are to analyze the current structure of accreditation and accreditation criteria in coaching because certain issues perceived to be solved by the Official College of Psychologists of Spain. Conducting a theoretical reflection on the subject, as to the structure of accreditation, is not well defined which represent areas of knowledge and intervention (specialty) versus sub-areas, areas of activity or intervention strategies (sub-specialties). With respect to accreditation in coaching, a variety of established criteria and a lack of consensus among the schools of psychologists are observed, in which, moreover, it is presented as something transverse and not related to specialties. As more remarkable, it is concluded that the structure of accreditation has to be configured with a first level with the title psychologist, a second level with professional specialty and a third level of expert, linked to the level 2. As accreditation coaching, the name of expert coaching psychologist (leve/ 3), a minimum of 100 hours of training and 100 hours of practice, overcome a personal interview and situational test, and renewal every 4 years.

\section{palabras clave/keywords:}

Colegios Oficiales de Psicólogos; Acreditación Profesional; Coaching.

Official Schools of Psychologists; Professional Accreditation; Coaching 


\section{Introducción}

Los colegios profesionales, corporaciones de derecho público, tienen como fines esenciales la ordenación del ejercicio de las profesiones, la representación exclusiva de las mismas, la defensa de los intereses profesionales y la protección de los intereses de los usuarios de los servicios de sus colegiados/as, en el que la certificación profesional de los psicólogos/ as (áreas y sub-áreas de conocimiento e intervención, ámbitos de actuación, estrategias de intervención, etc.) tiene un valor central en relación a estas cuestiones (Chacón, 2009).

En esta línea, el presente trabajo asume los objetivos de analizar la actual estructura de acreditación profesional (sistema y procedimiento) de los Colegios Oficiales de Psicología (COPs) de las diferentes Comunidades Autónomas de España (Madrid, Barcelona, Valencia, etc.) y del Consejo General de la Psicología de España (CGPE), así como los criterios establecidos por los COPs y el CGPE para la obtención de la acreditación en coaching dado su actual auge en los diferentes colegios y en la sociedad. Para ello, se ha realizado una reflexión teórica basada en artículos y documentos vinculados con las acreditaciones. A continuación, se aborda ambos temas debido a que, como se irá desarrollando en el presente trabajo, se observa algunas cuestiones a resolver por parte de las instancias colegiales.

\section{Estado de la cuestión}

\section{Estructura de la acreditación profesional.}

Desde el punto de vista de las competencias profesionales del psicólogo/a, la Psicología está presente en la mayoría de las actividades humanas como es en el ámbito de la salud, la educación, la empresa, el deporte, entre otras (COP, 1998; Peiró, 2010; Roe, 2003). La Psicología como disciplina básica genera distintas aplicaciones profesionales desde un tronco común de conocimiento pues las diferentes demandas sociales han modelado su perfil (Cantón, 2016; Gallegos, Berra, Benito, y López-López, 2014; Santolaya, Berdullas, y Fernández, 2001). La titulación profesional reconocida legalmente para ejercer en estos ámbitos es la de psicólogo/a, que en cuanto a su formación viene establecida por el acceso a la titulación de la Licenciatura o del Grado en Psicología, en la que además, se ha impuesto el modelo por área de conocimiento e intervención (especialidad), la cual requiere una formación específica complementaria (Fernández, 2017; Jarne, Vilalta, Arch, Guardia, y Pérez, 2012; Roe, 2003).

En relación a estas cuestiones, tanto en los COPs, con valor autonómico, como el CGPE, que tiene carácter nacional e integra a todos los COPs (CGPE, 2018a), existen ciertas acreditaciones vinculadas al ejercicio profesional del psicólogo/a. Por ejemplo, en el Colegio Oficial de Psicólogos de Madrid (COPM, 2018a) presentan acreditaciones nacionales de Experto en Psicología Educativa, Experto en Neuropsicología Clínica, Experto en Psicooncología y Psicología en Cuidados Paliativos, Experto en Psicología del Deporte, Experto en Psicología de Emergencias y Catástrofes y Experto en Psicología Aeronáutica, establecidas por las divisiones profesionales del CGPE (2018b), mientras que otras son propias de las secciones profesionales (ver COPM, 2018b) o grupos de trabajo (ver COPM, 2018c) del colegio autonómico, como son la Certificación Profesional como Psicólogo/a Mentor y/o Ges- 
tor en Programas de Mentoring, Psicólogo/a Experto en Psicología Forense, Psicólogo/a Experto en Salud Laboral y Psicólogo/a Experto en Coaching.

Para obtener la acreditación en alguna de las divisiones profesionales del CGPE, se requiere cumplir con unos requisitos básicos e indispensables como son estar en posesión del Grado o la Licenciatura en Psicología, o titulación homologada o declarada equivalente, estar colegiado/a en cualquiera de las modalidades existentes, estar al corriente del pago de los recibos correspondientes y no estar cumpliendo una sanción deontológica (CGPE, 2015). Además, se indica que existen unos requerimientos específicos como son tener un mínimo de 400 horas de formación especializada complementaria en el campo concreto de intervención a través de sistemas de formación reconocidos por el CGPE y al menos 4 años de experiencia profesional certificada. La renovación de la condición de acreditado se establece en 200 horas de formación cada 4 años.

Señalar que estos criterios específicos establecidos por el CGPE (2015) son orientativos y pueden variar ligeramente en función de la división en cuestión, como por ejemplo, la acreditación nacional del Psicólogo/a Experto en Neuropsicología Clínica que solicitan 500 horas de formación (CGPE, 2018c).

Partiendo de estos criterios, el reconocimiento de un área de conocimiento e intervención puede darse a dos niveles: oficial, en el que las administraciones públicas la reconocen legalmente bajo el término de Especialista (actualmente sólo existe la de Psicólogo/a Especialista en Psicología Clínica y Psicólogo/a General Sanitario) o por los COPs y el CGPE bajo el término Experto, con sustento en conceptos como el servicio a la sociedad, la garantía de calidad en la oferta profesional, el prestigio social, la lucha contra la intromisión y la formación continua, pero sin un reconocimiento legal (Chacón, 2009; Jarne et al., 2012; Santolaya et al., 2001). Estos autores señalan que si el ámbito de conocimiento e intervención está reconocido únicamente por los colegios no conlleva necesariamente consecuencias legales, pero las sociales pueden ser poderosas e influyentes, tanto para los colegiados/as como para la sociedad.

Además, como señalan diferentes trabajos (Arch, Cartil, Solé, Lerroux, Calderer, y PérezGonzález, 2013; Cantón, 2016; Chacón, 2009; Santolaya et al., 2001), es necesario la especialidad en las áreas de conocimiento e intervención como requisito previo al ejercicio profesional, ya que se ha observado que mejora el desempeño del psicólogo/a (e. g., adaptación al contexto de intervención, reducción de faltas en el código deontológico y eficacia de intervención).

También está la cuestión de las subespecialidades. La experiencia profesional demuestra que las competencias específicas de alto nivel que adquiere un/a psicólogo/a en su trabajo, pueden ir más allá de las propias de las especialidades, el cual está directamente relacionado con las demandas cambiantes del mercado laboral (Cantón, 2016; Peiró, 2010; Roe, 2003). Como indica Jarne et al. (2012), destacar que los modelos teóricos (psicoanálisis, orientación cognitiva-conductual, etc.), ámbitos de actuación (e. g., alto rendimiento y organizaciones en Psicología del Deporte) o estrategias de intervención (EMDR, hipnosis, reestructuración cognitiva, coaching, etc.) integran la subespecialidad y no la especialidad, ya 
que no configura áreas de competencia profesional. Estos autores destacan que se trata fundamentalmente de un tema técnico, por lo que cada colegio y/o división debe decidir si cree que debe existir y, si es así, que regule su reconocimiento de subespecialidad bajo el término de "Experto", etiqueta que se podría solapar con la que se utiliza en el área de conocimiento e intervención cuando no está legalmente reconocida, como se verá en la discusión.

\section{Acreditaciones en coaching}

Destacar que la aplicación del coaching está actualmente siendo ofertadas en el mercado laboral por una diversidad de profesiones (economistas, empresarios, preparadores físicos, abogados, etc.), entre ellos los psicólogos (COP, 2012). La actuación del psicólogo/a en el ámbito del coaching está en expansión y crecimiento, dando a la profesión del psicólogo/a una repercusión directa en imagen y prestigio en la sociedad.

A pesar de ello, el coaching es un tema de debate y en ocasiones de conflicto dentro y fuera de los COPs, en el que no se termina de concluir qué es el coaching, cuál es su origen o quién puede aplicarlo, entre otras cuestiones (García-Naveira, 2009; Grant, 2006; Useche, 2004). Sin ser el objeto central del presente trabajo, si se quiere delimitar algunos aspectos que están vinculados con la acreditación de coaching.

En primer lugar, cabe destacar que el psicólogo/a es profesional por ser psicólogo/a y no por la aplicación del coaching (García-Naveira, 2011, 2015), en el que no existe la profesión legal de coach ni centro de formación que habilite para su ejercicio profesional (INFOCOP, 2014).

Desde una perspectiva aplicada, en líneas generales, el coaching consiste en entrenar a una persona o grupo de ellas a través de conversaciones eficaces e intencionadas para movilizarla de manera exitosa hacia sus metas u objetivos (COPM, 2013). Técnicamente el coaching se define como una estrategia de intervención psicológica en modificación de la conducta, ya que su aplicación produce cambios mantenidos en la cognición, emoción y conducta de la persona (Cantón, 2014; García-Naveira, 2011; Grant, 2006), así como la mejora del bienestar y la salud psicológica del mismo, tanto en adolescentes como adultos (García-Naveira, 2016; García-Naveira, García-Mas, Ruiz-Barquín, y Cantón, 2017).

Para su aplicación, se requiere el aprendizaje o desarrollo de determinadas habilidades de comunicación (e. g., la escucha empática, el realizar preguntas eficaces, el silencio y dar feedback), la utilización de unos principios y postulados (e. g., acompañamiento no directivo basado en el diálogo socrático y en el autodescubrimiento inducido) y el seguimiento de unas fases o etapas dentro de un proceso de coaching (e. g., establecer el vínculo y la alianza, definir la situación actual, la situación ideal, el plan de acción y el seguimiento), entre otras cuestiones (García-Naveira, 2013, 2015; García-Naveira y Ruiz-Barquín, 2014).

Ante este panorama y la demanda social en torno al coaching, el COP (2012) estableció inicialmente unos criterios para que los COPs interesados pudieran desarrollar sus propias acreditaciones en esta estrategia de intervención psicológica. Prueba de ello es que el Colegio Oficial de Psicólogos de Andalucía Occidental (COPAO, 2014), Colegio Oficial de Psicólogos de Cataluña (COPC, 2014), Colegio Oficial de Psicólogos de la Comunidad Valenciana (COPCV, 2013), Colegio Oficial de la Psicología de las Islas Baleares (COPIB, 2017), 
Colegio Oficial de Psicólogos de Madrid (COPM, 2013) y Colegio Oficial de Psicólogos de la Región de Murcia (COPRM, 2016), han creado y puesto en marcha una acreditación para el empleo experto del coaching en sus correspondientes comunidades autónomas.

Con el objetivo de analizar los criterios de acreditación del coaching de cada uno de los colegios profesionales que lo ofertan, a continuación se presenta un resumen de los aspectos más destacables, como son: la denominación de la acreditación de cada una de ellas, los requisitos formales básicos que solicitan, las horas de formación, las horas prácticas, niveles de acreditación, otros méritos solicitados, los procesos de evaluación, la constitución del comité evaluador y los criterios de la renovación.

En cuanto a la denominación de la acreditación, los términos que aparecen son los de "profesional" con una frecuencia de 5 sobre 7 acreditaciones (COP, 2012; COPAO, 2014; COPCV, 2013; COPIB, 2017; COPM, 2013), "coaching" con una frecuencia de 5 sobre 7 acreditaciones (COP, 2012; COPC, 2014; COPCV, 2013; COPIB, 2017; COPM, 2013), "experto" con una frecuencia de 4 sobre 7 acreditaciones (COP, 2012; COPC, 2014; COPIB, 2017; COPM, 2013), "psicólogo" con una frecuencia de 4 sobre 7 acreditaciones (COP, 2012; COPCV, 2013; COPIB, 2017; COPM, 2013), "psicólogo/a coach" con una frecuencia de 2 sobre 7 acreditaciones (COPAO, 2014; COPRM, 2016), "Psicología del coaching" con una frecuencia de 1 sobre 7 acreditaciones (COPC, 2014) y "especialista" con una frecuencia de 1 sobre 7 acreditaciones (COPCV, 2013).

Respecto a los requisitos formales básicos para acceder a la acreditación, los 7 colegios profesionales indican que hay que tener la Licenciatura o Grado de Psicología, estar colegiado y al corriente de la cuota colegial y no tener sanción deontológica (COP, 2012; COPAO, 2014; COPC, 2014; COPCV, 2013; COPIB, 2017; COPM, 2013; COPRM, 2016).

En relación a las horas de formación en coaching, con una frecuencia de 3 sobre 7 acreditaciones diferencian entre las horas de formación acreditadas por Entidades Reconocidas por el Colegio (ERC) y las Realizadas en el Propio Colegio -RPC- (COP, 2012; COPC, 2014; COPIB, 2017), mientras que 4 sobre 7 acreditaciones destacan las horas de formación a nivel genérico sin distinciones (COPAO, 2014; COPCV, 2013; COPM, 2013; COPRM, 2016). En concreto, el COP, COPC y COPIB indican 100 horas de ERC o 50 horas en el RPC, mientras que el COPRM lo establecen en 100 horas sin esa distinción, el COPM en 120 horas, el COPCV en 130 horas y el COPAO en 150 horas. También destacar que en el COPCV incluye adicionalmente 10 horas de formación en la aplicación del código deontológico en coaching.

En cuanto a los niveles de acreditación y horas prácticas, con una frecuencia de 6 sobre 7 acreditaciones define un único nivel (COP, 2012; COPC, 2014; COPCV, 2013; COPIB, 2017; COPM, 2013; COPRM, 2016) mientras que la acreditación restante establece 3 niveles (COPAO, 2014). En concreto, dentro del único nivel, con una frecuencia de 6 sobre 6 acreditaciones se solicita 100 horas de prácticas (COP, 2012; COPC, 2014; COPCV, 2013; COPIB, 2017; COPM, 2013; COPRM, 2016), en el que además el COPM pide 5 sesiones supervisadas por un Psicólogo Experto en Coaching, mientras que el COPRM solicita 12 horas de supervisión en un mínimo de 4 sesiones. 
En el caso del COPAO (2014) las horas prácticas varían en función del nivel (desde 60 horas hasta 400 horas prácticas), al igual que el número de procesos solicitados (desde 5 hasta 30), horas remuneradas (desde 20 hasta 300) y horas de supervisión (desde 15 hasta 50).

Como alternativa, con una frecuencia de 4 sobre 7 acreditaciones presentan la opción de superar una prueba situacional (COP, 2012; COPC, 2013; COPCV, 2013; COPIB, 2017). También el COPC (2014) y COPIB (2017) proporcionan otras posibilidades para cubrir este requisito como es el poseer el certificado en los niveles de rango superior de certificación en asociaciones profesionales en coaching (AECOP, EMCC y ICF) o especialización internacional en Coaching Psycology según ISCP.

Respecto a otros méritos, con una frecuencia de 4 sobre 7 acreditaciones se solicita la elaboración de un ensayo, exponer un caso práctico o trabajo de investigación en relación al coaching (COPCV, 2013; COPAO, 2014; COPM, 2013; COPRM, 2016), aunque en el caso del COPAO (2014) sería para el nivel 2 y 3 . Por su parte, con una frecuencia de 3 sobre 7 acreditaciones no solicitan ninguna documentación adicional (COP, 2012; COPC, 2014; COPIB, 2017).

En relación con la evaluación del psicólogo solicitante de la acreditación, con una frecuencia de 5 sobre 7 acreditaciones se requiere superar una entrevista con la comisión de expertos (COP, 2012; COPAO, 2014; COPC, 2014; COPCV, 2013; COPIB, 2017) o con una frecuencia de 2 sobre 7 acreditaciones tener 4 sesiones de coaching supervisada por un Psicólogo Experto en Coaching (COP, 2012; COPCV, 2013) o recibir 6 sesiones de coaching y tener 3 procesos supervisados de coaching (COPIB, 2017), mientras que el COPM (2013) y COPRM (2016) sería suficiente cumplir con los requisitos generales y específicos expuestos con anterioridad, en el que en este último caso también se piden 2 cartas de propuesta de 2 psicólogos/as coach acreditados por el COPRM.

En cuanto a la composición del comité evaluador, en el COPAO (2014) lo integran 5 evaluadores ( 3 pertenecen al grupo de trabajo en coaching y 2 a la Universidad de Sevilla); en el COPC (2014) y COPIB (2017) presentan a 4 evaluadores (1 miembro de la junta de gobierno y 3 miembros de la sección de Psicología y coaching) aunque en caso necesario se puede recurrir a un experto/a en coaching del colegio; en el COPM (2013) lo conforman 4 evaluadores ( 1 representante de la acreditado en coaching de la sección de Psicología del Trabajo, Organizaciones y RRHH y 2 psicólogos acreditados en coaching de prestigio y 1 miembro de la junta de gobierno); el COPCV (2013) lo integra un comité técnico designado por la junta de gobierno asesorada por psicólogos/as de reconocido prestigio en el ámbito del coaching; y por último, el COPRM (2016) está constituido por 4 evaluadores (1 miembro de la junta de gobierno y 3 psicólogos/as coach acreditados).

Para la duración de la validez de la acreditación el COPAO (2014), COPIB, (2017), COPM (2013) y COPMRM (2016) señalan que será de 4 años, mientras que el COP (2012), COPC (2014) y COPCV (2013) lo establecen en 5 años. Los interesados en la renovación tendrán que acreditar 30 horas supervisadas de coaching (COPAO, 2014), 40 horas de formación continua en coaching y 50 horas de supervisión por un Psicólogo/a Experto/a en Coaching (COP, 2012; COPC, 2014; COPCV, 2013; COPM, 2013) o 40 horas de formación 
continua en coaching, 2 procesos de coaching al año y 1 supervisión de coaching al año (COPIB, 2017), mientras que el COPRM se requiere 10 horas de formación y 10 horas de supervisión al año.

\section{Discusión}

Continuando con los apartados del presente trabajo, a continuación se discute sobre la estructura de acreditación y los criterios de acreditación en coaching de los colegios de psicología.

\section{Estructura de acreditación}

Como indican diferentes autores (Arch et al., 2013; Cantón, 2016; Fernández, 2017; Gallegos et al., 2014; Jarne et al., 2012; Roe, 2003; Santolaya et al., 2001), se necesita la especialización en un área de conocimiento e intervención para un correcto desempeño profesional del psicólogo/a. Para ello, se requiere tener una estructura coherente y articula para conseguir dicha especialización, sin confundir con las posibles competencias específicas aprendidas que entrarían dentro de la sub-especialización.

Siguiendo con lo expuesto, la estructura que define la competencia y acreditación profesional en Psicología tiene que quedar configurada en forma piramidal invertida, en el que en el primer nivel se sitúa el título de psicólogo/a como unidad funcional de la Psicología, un segundo nivel con las especialidad profesional correspondiente a las diferentes áreas de conocimiento e intervención y un tercer nivel de experto relacionado con las competencias específicas adquiridas (sub-área, ámbito o estrategia de intervención). Por ejemplo, Psicólogo Especialista en Psicología del Deporte Experto en Alto Rendimiento, Psicólogo Especialista en Psicología Clínica Experto en Intervención con Niños y Adolescentes, Especialista en Psicología de Emergencias y Catástrofes Experto en EMDR, etc.

Esta nomenclatura de "especialista" se solaparía con la de Psicología Clínica, única legalmente reconocida como tal. Actualmente, las acreditaciones se denominan como "experto", no diferenciándose las que son de la especialidad o las que son de experto propiamente dichas (sub-especialidades). Esto puede dar lugar a una mayor confusión terminológica y no técnica entre los colegiados/as y la sociedad. Por ello, como propuesta, las especialidades se tendrían que denominar como "especialista" y no "experto" más allá de su actual valor legal. Por ejemplo, Especialista en Psicología del Deporte vs. Experto en Psicología del Deporte.

Los requisitos para la especialización en el área de conocimiento e intervención (especialidad) están bien establecidos por parte del CGPE (2015), aunque quedaría pendiente el definir cuáles son las acreditaciones en el nivel de experto (sub-especialidad), las horas de formación necesarias, duración de la misma y los criterios de renovación, entre otros temas. Como indican Jarne et al. (2012), estas cuestiones tendrían que ser estudiadas y definidas por la Comisión Nacional de Acreditación Profesional (CNAP) del CGPE.

Otro punto asociado que debería abordar la CNAP es que existen acreditaciones a nivel autonómico que no están establecidas a nivel nacional. Ambas deberían de estar alineadas y existir un único criterio a nivel nacional. En este sentido, la organización colegial debería 
establecer un procedimiento marco, que evite conflictos o duplicidades, para que sea gestionado por el CGPE y/o COPs, que atenderán a las especificidades de cada área o sub-área. En el caso de que la acreditación sea nueva o novedosa, el colegio autonómico en cuestión podrá proponer al CGPE su creación o viceversa, buscando la unidad colegial.

\section{Acreditaciones en coaching}

En primer lugar, continuando con la estructura de acreditación previamente establecida, al revisar las diferentes acreditaciones, se observa que el coaching no se presenta como un nivel de experto (sub-especialidad), sino como algo transversal y dentro de la capacitación general psicológica, no vinculado a una única área de conocimiento e intervención (especialidad). Según este planteamiento, por ejemplo, el colegiado/a debería de ser Especialista en Psicología de la Actividad Física y del Deporte (área de conocimiento e intervención) y Experto en coaching (sub-área, en este caso como estrategia de intervención psicológica en modificación de la conducta).

Por otro lado, se observa una variedad de criterios y falta de unidad institucional entre los colegios profesionales en cuanto a la acreditación en coaching. Lo único que comparten dichas acreditaciones son los requerimientos formales de tener la Licenciatura o Grado en Psicología, estar colegiado y al corriente del pago y no tener sanción deontológica.

Respecto a la denominación de la acreditación, surgen los términos de "profesional", “coaching”, "experto", "psicólogo", "psicólogo/a coach", "Psicología del coaching” y "especialista". Dentro de esta variedad de términos según los criterios previamente establecidos, la denominación correcta tendría que ser la de "Psicólogo Experto en Coaching" como sub-especialidad.

En cuanto a las horas de formación existe un rango que varía desde las 100 horas hasta las 150 horas. Además, existen diferencias en algunos colegios entre las horas de formación que se requiere si el curso proviene del propio colegio (se reduciría a 50 horas) o de una entidad externa (las establecidas con anterioridad).

Con el objetivo de esclarecer esta cuestión, se requeriría definir en general las horas de formación y experiencia práctica necesaria para acceder a un nivel de experto tras la obtención de la especialización y, en concreto, establecer un temario con una carga lectiva en coaching (teórico-práctico) compartida entre los colegios como bloque genérico. Además, como señalan García-Naveira y Ruiz-Barquín (2014), se necesita atender las características de cada área de conocimiento e intervención, aspecto que tendría que estar presente en la formación como bloque específico (e. g., aplicación del coaching en deportistas para los psicólogos Especialistas en Psicología del Deporte).

Partiendo de estas cuestiones y analizando las acreditaciones existentes, aspecto que quedaría abierto al debate técnico, como propuesta y contribución a esta cuestión, posiblemente con 100 horas de formación sería suficiente como condición mínima para la acreditación del nivel de experto, como puede ser el caso del coaching, aspecto que quedó establecido por el COP (2012) como referente nacional de todos los colegios autonómicos y reforzada por el COPC (2014), COPIB (2017) y el COPRM (2016). 
En lo que se refiere a la distinción entre la formación del propio colegio y otras entidades, indicar que si la entidad es externa y reconocida por el colegio, se presupone que está validado el contenido, la metodología, el profesorado y el estándar de calidad de los cursos, por lo que no sería necesario realizar dicha distinción.

En relación a los niveles de acreditación y horas prácticas, se podría tomar como referencia un único nivel y el mínimo de 100 horas prácticas certificadas que establece el COP (2012) y la mayoría de los colegios (COPC, 2014; COPCV, 2013; COPIB, 2017; COPM, 2013; COPRM, 2016), entendiendo que esta cantidad es suficiente debido a que el psicólogo/a ya posee la mayoría de las competencias y conocimientos propios del coaching (García-Naveira, 2013; García-Naveira et al., 2016, 2017).

En cuanto a que determinadas horas (30) sean remuneradas, tal y como solicita algunos colegios (COPAO, 2014; el COPC, 2014; COPIB, 2017; COPRM, 2013), con el objetivo de que haya un acceso a la acreditación para todos los colegiados/as interesados/as, debido a que lo que se está acreditando son horas de prácticas y las competencias adquiridas, y a la situación económica y dificultad del mercado laboral actual en España, se requiere establecer un procedimiento de regulación para aquellas personas que no pueden justificar que determinadas horas de desempeño hayan sido remuneradas, o en su defecto, directamente eliminar este requisito.

Otra cuestión a definir es la supervisión en coaching (4-5 sesiones), en la línea establecida por algunos colegios (COP, 2012; COPAO, 2014; COPM, 2013; COPCV; COPRM, 2016). Un programa de formación en coaching requiere una supervisión del alumno/a respecto a su aplicación (García-Naveira, 2015), por lo que una vez establecido, no sería necesario una supervisión posterior en las horas prácticas para la obtención de la acreditación, en el que también se tiene que evitar que termine siendo un negocio y se especule con este requisito (e. g., cobrar por una supervisión). Es por ello que, como se cita en párrafos posteriores, habría que centrar los esfuerzos en superar una entrevista personal y prueba situacional que garantice un estándar de calidad en la aplicación del coaching.

En cuanto a otros méritos que se pueda requerir para obtener la acreditación, partiendo del COP (2012) y de determinados colegios (COPC, 2014; COPIB, 2017), no sería una cuestión central, por lo que no se requeriría la realización de un ensayo, exposición de un caso práctico o trabajo de investigación sobre coaching, cuestiones que tal vez si se podría integrar en los cursos formativos.

Respecto a la evaluación de los candidatos, siguiendo con lo establecido por el COP (2012) y algunos colegios (COPAO, 2014; COPC, 2014; COPCV, 2013; COPIB, 2017), debido a que se busca evaluar una competencia aprendida en coaching, se debería de realizar una entrevista personal y superar una prueba situacional, adaptando esta circunstancia a las nuevas tecnologías y la superación de las barreras geográficas (e. g., online, vídeo u otro procedimiento).

En relación al comité evaluador, debido a que el COP (2012) no especifica quien lo inte- 
graría y la variedad de integrantes, criterios y posicionamientos que presentan los colegios, una posible alternativa puede ser adaptar las indicaciones de constitución del comité de evaluación de las acreditaciones de especialistas (CGPE, 2015), el cual estaría establecido por 5 evaluadores, como por ejemplo, 1 miembro designado por la junta de gobierno, 2 integrantes de la división, sección o grupo de trabajo específico (e.g., Psicología del Deporte) y 2 psicólogos expertos en coaching de prestigio, preferentemente del ámbito concreto de intervención.

En cuanto a la duración de la validez de la acreditación podría quedar establecida en 4 años como indican varios colegios (COPAO, 2014; COPIB, 2017; COPM, 2013; COPMRM, 2016), contrario a lo que define el COP (2012) y otros colegios (COPC, 2014; COPCV, 2013), ya que esta no debería de ser superior a la duración de la acreditación de la especialidad, que se define en 4 años (CGPE, 2015).

En cuanto a la renovación, se entiende que si el psicólogo/a obtiene la acreditación de experto en coaching, este ya posee las competencias básicas necesarias para aplicarlo, por lo que sería razonable, como indica el COP (2012) y algunos colegios (COPC, 2014; COPCV, 2013 y COPM, 2013), certificar 40 horas de formación, 50 horas prácticas en coaching, una entrevista personal y superar una prueba situacional, como sucede en la obtención de la acreditación.

Otra cuestión a atender en la obtención de las horas de formación y prácticas para la renovación del candidato/a, es la de contabilizar la impartición de cursos y realización de publicaciones de artículos científicos/aplicados en coaching, tal y como sucede en las acreditaciones nacionales (CGPE, 2018b).

Otra limitación a solventar en un futuro, pese a la relevancia que tiene las competencias profesionales en los procesos actuales de acreditación de las especialidades y sub-especialidades de la psicología (Peiró, 2010), es la de integrar un sistema de evaluación por competencias, cuestión que no se observa en la actual acreditación de coaching.

Y por último, tampoco se hace referencias a la situación de estas cuestiones fuera de España y/o en relación con aspectos formativos formales, lo que permitiría ubicar mejor su planteamiento, aspectos a estudiar en futuros trabajos.

En la Tabla 1, se presenta un resumen y propuesta de los aspectos anteriormente citados, en el cual se integra tanto la estructura de acreditación como los criterios de acreditación en coaching.

Para terminar, dado que el soporte institucional ha jugado un rol central en la evolución de la Psicología, será necesario afianzar o redefinir, según sea el caso, los objetivos, criterios, procesos y acciones de los colegios de psicología en torno a las acreditaciones, especialmente en el coaching. Llegar a alcanzar acuerdos sobre estas cuestiones representa un gran reto, ya que requiere cambiar los sistemas y prácticas existentes, y hay que superar los intereses establecidos, aspectos que necesitan ser solucionados a corto o medio plazo. 
Tabla 1. Resumen y propuesta de estructura de acreditación y criterios para obtener la acreditación de Psicólogo/a Experto/a en Coaching.

$\begin{array}{ll}\begin{array}{l}\text { Requisitos formales } \\ \text { básicos }\end{array} & \begin{array}{l}\text { Nivel 1: poseer la Licenciatura o Grado en Psicología, estar } \\ \text { colegiado/a y al corriente de la cuota colegial y no tener } \\ \text { sanción deontológica. }\end{array}\end{array}$

\begin{tabular}{ll}
\hline $\begin{array}{l}\text { Requisitos formales } \\
\text { específicos }\end{array}$ & $\begin{array}{l}\text { Nivel 2: tener la acreditación de especialista en un área } \\
\text { de intervención: 400 horas de formación y } 4 \text { años de } \\
\text { experiencia profesional. } \\
\text { Renovación: 200 horas de formación cada 4 años }\end{array}$ \\
\hline $\begin{array}{l}\text { Denominación de la } \\
\text { acreditación }\end{array}$ & Nivel 3: Psicólogo/a Experto en Coaching \\
\hline Horas de formación & $\begin{array}{l}\text { Mínimo 100 horas de formación impartidas por los COPs o } \\
\text { entidades reconocidas por las mismas. }\end{array}$ \\
\hline $\begin{array}{l}\text { Niveles de } \\
\text { acreditación y horas } \\
\text { prácticas. }\end{array}$ & $\begin{array}{l}\text { Único nivel con un mínimo de 100 horas prácticas } \\
\text { certificadas. }\end{array}$ \\
\hline
\end{tabular}

Otros méritos No se requiere

\begin{tabular}{ll}
\hline Evaluación & $\begin{array}{l}\text { Certificar formación y horas prácticas establecidas } \\
\text { Entrevista personal y prueba situacional (presencial, online, } \\
\text { vídeo, etc.) }\end{array}$ \\
\hline Comité evaluador & 5 miembros: 1 miembro designado por la Junta de \\
& $\begin{array}{l}\text { Gobierno, } 2 \text { integrantes de la división, sección o grupo de } \\
\text { trabajo específico y } 2 \text { psicólogos/as expertos en coaching de } \\
\text { prestigio preferentemente del área en cuestión }\end{array}$ \\
\hline Renovación & Cada 4 años \\
& 40 horas de formación impartidas por los COPs o entidades \\
& reconocidas para ello. \\
& 50 horas prácticas certificadas no necesariamente \\
& remuneradas. \\
& Entrevista personal y prueba situacional (presencial online, \\
& vídeo, etc.).
\end{tabular}




\section{Referencias}

Arch, M., Cartil, C., Solé, P., Lerroux, V., Calderer, N., y Pérez-González, A. (2013). Deontología profesional en Psicología: estudio descriptivo de las denuncias atendidas por la comisión deontológica del COPCAT. Papeles del Psicólogo, 34 (3), 182-189.

Cantón, E. (2014). ¿El coaching es psicología? ¿Quién puede aplicar el coaching y en qué entornos? Informació Psicologica, $107,2-10$.

Cantón, E. (2016). La especialidad profesional en Psicología del Deporte. Revista de Psicología aplicada al Deporte y al Ejercicio Físico, 1(e2), 1-12. DOI: 10.5093/rpadef2016a2

Chacón, F. (2009). La formación en los colegios profesionales, el aprendizaje a lo largo de la vida, la acreditación profesional y la calidad de... ARBOR Ciencia, Pensamiento y Cultura, 185 (Extra), 111-124. D0I: 10.3989/ arbor.2009.extran1209

Colegio Oficial de Psicología de Andalucía Occidental (COPA0, 2014). Procedimiento de acreditación profesional del psicológo/a coach. COPAO: Sevilla

Colegio Oficial de Psicólogos de Cataluña (COPC, 2014). Acreditació de I'expertesa en psicologia coaching. COPC: Barcelona.

Colegio Oficial de Psicólogos de la Comunidad Valenciana (COPCV, 2013). Acreditación profesional como psicólogo especialista en coaching. COPCV: Valencia.

Colegio Oficial de Psicólogos de España (COP, 1998). Perfiles profesionales del psicólogo. COP: Madrid.

Colegio Oficial de Psicólogos de España (COP, 2012). Criterios de acreditación para el ejercicio profesional del psicólogo experto en coaching. COP: Madrid.

Colegio Oficial de la Psicología de las Islas Baleares (COPIB, 2017). Acreditación profesional de psicólogo/a experto/a en coaching. Disponible en http://www.copib.es/intranet/files/01_09_1610_42_091_PsEC_acreditacion.pdf

Colegio Oficial de Psicólogos de Madrid (COPM, 2013). Acreditación profesional como psicólogo experto en coaching. COPM: Madrid

Colegio Oficial de Psicólogos de Madrid (COPM, 2018a). Acreditaciones Profesionales. Disponible en http://www. copmadrid.org/web/colegiados/acreditaciones-profesionales

Colegio Oficial de Psicólogos de Madrid (COPM, 2018b). Secciones del colegio. Disponible en http://www.copmadrid. org/web/el-colegio/secciones-colegio

Colegio Oficial de Psicólogos de Madrid (COPM, 2018c). Grupos de trabajo. Disponible en http://www.copmadrid.org/ web/colegiados/grupos-de-trabajo?red=9

Colegio Oficial de Psicólogos de la Región de Murcia (COPRM, 2016). Acreditación de psicólogo/a coach. COPRM: Murcia.

Consejo General de la Psicología de España (CGPE, 2015). Reglamento marco de las divisiones profesionales. CGPE: Madrid.

Consejo General de la Psicología de España (CGPE, 2018a). Colegios Oficiales de Psicólogos. Disponible en https:// www.cop.es/index.php

Consejo General de la Psicología de España (CGPE, 2018b). Comisión nacional de acreditación profesional. Disponible en http://www.acreditaciones.cop.es/

Consejo General de la Psicología de España (2018c). Acreditación nacional del psicólogo/a experto/a en neuropsicología clínica. Disponible en http://www.acreditaciones.cop.es/pdf/APNeuropsicologia.pdf

Fernández, J. R. (2017). Cambiar para avanzar. Un análisis del debate sobre itinerario formativo de la Psicología Clínica en España. Papeles del Psicólogo, 38(2), 81-93. 
Gallegos, M., Berra, M., Benito, E., y López López, W. (2014). Las nuevas dinámicas del conocimiento científico y su impacto en la Psicología Latinoamericana. Psicoperspectivas, 13(3), 106-117.

García-Naveira, A. (2009). Psicología y coaching: ¿reflexión y acción? XII Congreso Andaluz de Psicología del Deporte y de la Actividad Física. Jaén.

García-Naveira, A. (2011). Aproximación al empleo profesional del coaching en el deporte. Informació Psicológica, 101, 26-39.

García-Naveira, A. (2013). Aplicación profesional del coaching en el deporte: un estudio de caso único. Cuadernos de Psicología del Deporte, 13(2), 101-112.

García-Naveira, A. (2015). Aplicación profesional del coaching en el deporte. En A., García-Naveira y L., Locatelli, Avances en Psicología del Deporte (pp. 121-144). Barcelona: PaidoTribo.

García-Naveira, A. (2016). Percepción del bienestar y de la salud psicológica, y la eficacia de un programa de intervención en coaching en deportistas de rendimiento. Revista Iberoamericana de Psicología del Ejercicio y el Deporte, 11(2), 211-219.

García-Naveira, A., García-Mas, A., Ruiz-Barquín, R., y Cantón, E. (2017). Programa de intervención basada en el coaching en jóvenes deportistas de alto rendimiento, y su relación con la percepción de bienestar y salud psicológica. Revista de Psicología del Deporte, 26(2), 37-44.

García-Naveira, A. y Ruiz-Barquín, R. (2014). Liderazgo y coaching deportivo. Madrid: Síntesis.

Grant, A. (2006). A personal perspective on professional coaching and the development of coaching psychology. International Coaching Psychology Review, 1(1), 12-19.

INFOCOP (2014). La división de Psicología del Deporte del COP se pone en contacto con el Ministerio para aclarar dudas sobre el coaching. Disponible en http://www.infocop.es/view_article.asp?id=5183

Jarne, A., Vilalta, R., Arch, M., Guardia, J., y Pérez, A. (2012). Especialidades y acreditaciones en Psicología. Papeles del Psicólogo. 33(2), 90-100

Peiró, J. M. (2010). Competencias para el ejercicio profesional del psicólogo. Formación continua a distancia. COP: Madrid.

Roe, R. (2003). ¿Qué hace competente a un psicólogo? Papeles del Psicólogo, 86, 1-12.

Santolaya, F., Berdullas, M., y Fernández, J. (2001). The Decade 1989-1998 in Spanish Psychology: An Analysis of Development of Professional Psychology in Spain. The Spanish Journal of Psychology, 4(2), 237-252.

Useche, M. C. (2004). El coaching desde una perspectiva epistemológica. Revista de Ciencias Sociales, 105(3), 125-132. 